\title{
Magnetism of purified amorphous carbon
}

\author{
Anton Komlev ${ }^{1,2 *}$, Erkki Lähderanta ${ }^{1}$, Evgeniy Shevchenko $^{3}$ and Nikolay Vorob'ev-Desyatovskii ${ }^{4}$ \\ ${ }^{1}$ Lappeenranta University of Technology, School of Engineering Science, 53850 Lappeenranta, Finland \\ 2 Saint-Petersburg State Electrotechnical University “LETI”, 197376 Saint-Petersburg, Russia \\ ${ }^{3}$ Saint-Petersburg State University, Research Park, 199034 Saint-Petersburg, Russia \\ ${ }^{4}$ JSC Polymetal Engineering, Hydrometallurgical Department, 198216 Saint-Petersburg, Russia
}

\begin{abstract}
In this work, magnetic properties of activated carbon coal, which is also named amorphous carbon, were investigated. Composition and structure analysis was performed by XRD, SEM and EDS techniques. Position and width of peaks on XRD pattern confirmed amorphous structure of coals with small degree of graphitization. Magnetic measurements were performed by SQUID magnetometer. According to thermomagnetic measurements, in part of the samples, antiferromagnetic transition was observed near $120 \mathrm{~K}$. Nature of such phenomena, as well as effect of oxygen and magnetic contaminations on thermomagnetic measurements are discussed referring to similar results from previous researches and data from literature.
\end{abstract}

\section{Introduction}

From ancient times carbon materials are widely used for many applications. After recent discoveries of fullerenes, carbon nanotubes and graphene, carbon-based materials became one of the most promising and universal materials. Among a variety of their outstanding mechanical, conductive and many other characteristics [1], also exists a number of controversial topics. One of such problematic areas is possibility of making carbon materials magnetic.

Despite the decades of investigations, magnetic properties of carbon allotropes is still a debatable topic. While the defect-induced paramagnetic behaviour is acknowledged, the problem of possibility of magnetic ordering in carbon materials is not solved yet. Many researches have demonstrated impurity-free carbon materials, where spontaneous magnetization was observed (e.g. Ref. [2, 3]). Such possibility for carbon materials is also predicted by many theoretical works (e.g. Ref [4]), meaning that magnetic carbon does not confront to general principles of magnetism. However, the question of intrinsic or extrinsic character and problem of the reproducibility of such results exists thus far.

In this work, thermomagnetic behavior of activated carbon coal was investigated. This material is also named amorphous carbon and is widely used on practice for extraction of noble metals from ores and for filtration processes. Amorphous carbon can be considered as many disorder graphite planes of different size and with big amount of defects. Thus, if carbon-based magnetism is promoted by defects, there should be a possibility to observe it in such structures. Investigation of magnetic properties of coals is important from both theoretical and practical points of view: first of all, it will give a contribution to the discussions about carbon-based magnetism; secondly, understanding of magnetic behavior of this material is essential since some efforts are undertaken to produce magnetic filtering material based on coals $[5,6]$.

\section{Materials and Methods}

Commercially available coal Goldcarb WSC208C-GR produced from coconut shells and provided by Chemviron Carbon company was used as a raw material. Activation of the raw material was done by ball-milling. To remove the acid-soluble ash content, coal was washed with $1 \mathrm{M}$ solution of $\mathrm{HClO}_{4}$ for one day, after what it was washed with distilled water for three weeks in Soxhlet extractor. Water in the extractor was changed every day and the conductivity of the water was checked to detect any contaminations, removed from coal. Part of the samples was saturated by copper complexes from an aqueous solution of $\left(\mathrm{Na}\left[\mathrm{Cu}(\mathrm{CN})_{2}\right]\right)$. Motivation for such modification was a prior research related to absorption of a copper complexes during extracting of gold from cyanide solutions, which will not be discussed here. Part of both clean and modified samples was additionally dehydrated at $80^{\circ} \mathrm{C}$ temperature and 10 mbar vacuum before magnetic measurements.

Structure and composition of the samples were analyzed by means of scanning electron microscope (SEM) Hitachi SU3500 with built-in energy-dispersive Xray spectroscopy (EDS) radiation and $\mathrm{X}$-ray diffraction (XRD) device Bruker D8 Advance using $\mathrm{Cu} \mathrm{K \alpha}$ $(\lambda=1.5418 \AA)$. Magnetic characterization was performed on a superconducting quantum interference device (SQUID) Quantum Design MPMS-XL-1 SQUID magnetometer with an up to $1 \mathrm{~T}$ magnet and working temperatures range of $1.8-400 \mathrm{~K}$.

${ }^{*}$ Corresponding author: KomlevAnton@hotmail.com 

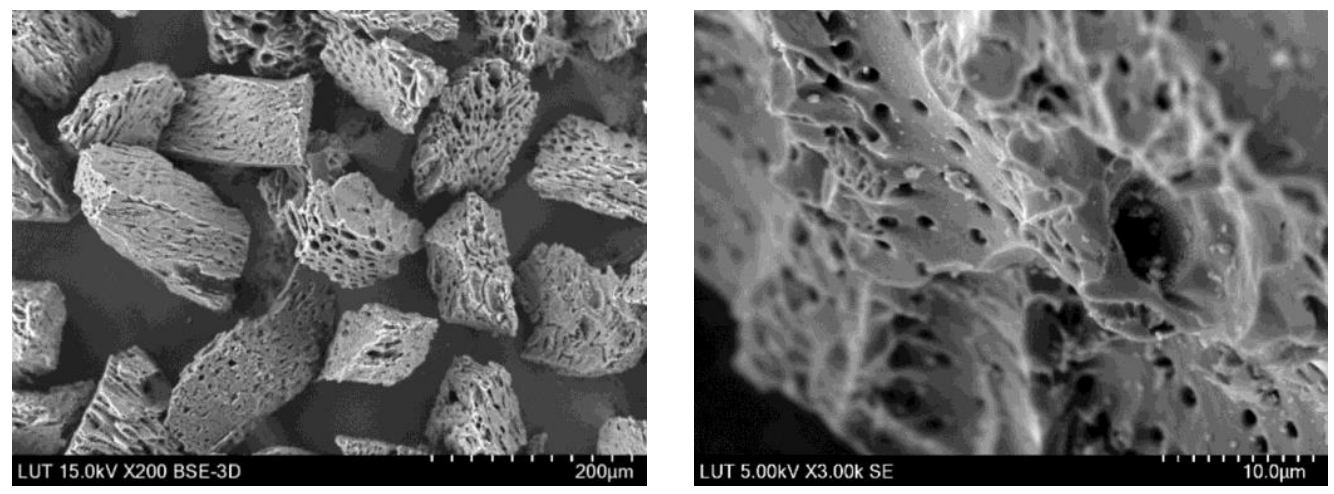

Fig. 1. SEM images of carbon coal, demonstrating high porosity of the samples: $\times 200$ magnification on the left and $\times 3000$ on the right pictures.

\section{Results and discussion}

According to SEM images on Fig. 1, after activation and purification, the material consists of particles of $\sim 100 \div 200 \mu \mathrm{m}$ size. These particles demonstrate highly developed surface and structure of the pores. Theirs size varies from $\sim 15 \mu \mathrm{m}$ to less than $100 \mathrm{~nm}$, what is a characteristic of typical activated carbon. Big surface area and, thus, big amount of edge states is essential and desirable for both adsorption processes and possible magnetic ordering due to edge-states magnetism.

Structure of the coal was also examined by XRD technique (Fig. 2). The XRD pattern demonstrated two broad peaks at $2 \theta \approx 25^{\circ}$ and $2 \theta \approx 45^{\circ}$, which correspond to reflection from graphite (002) and (101) planes respectively. High ratio of (101) peak intensity to (002) can be an indicator of very high porosity of the sample [7]. Overall, such pattern is typical for disordered carbon structures with small degree of graphitization, meaning that in this material exists some disordered graphite planes, but it is not completely amorphous carbon [8].

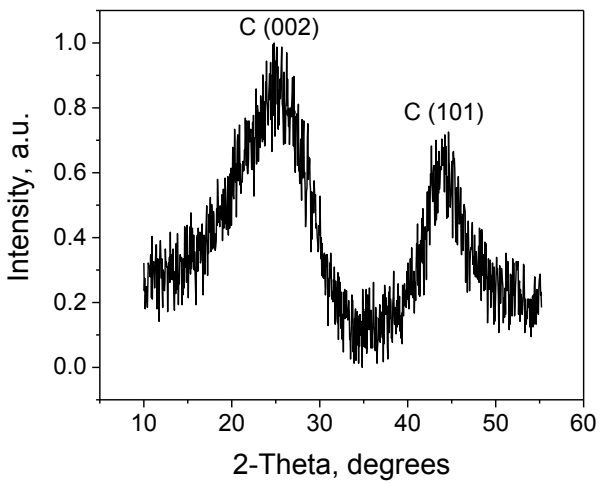

Fig. 2. XRD pattern of purified activated carbon coal.

Composition analysis of clean coal, performed by EDS with $20 \mathrm{kV}$ accelerating voltage, showed $300 \mathrm{ppm}$ of both $\mathrm{S}$ and $\mathrm{Al}$. For modified with copper samples, in addition to the same amount of $\mathrm{S}$ and $\mathrm{Al}, 300 \mathrm{ppm}$ of $\mathrm{Cl}$ and $3000 \mathrm{ppm}$ of $\mathrm{Cu}$ were detected. Precise numbers are not crucial here, because the most important result is absence of any magnetic impurities (e.g. Fe, Co, Ni).
However, it has to be mentioned, that in the similarly prepared batch of samples, EDS analysis performed on EDX-8000 (Shimadzu) in other laboratory detected additional contaminations: $350 \mathrm{ppm}$ of $\mathrm{Si}, 50 \mathrm{ppm}$ of $\mathrm{Ca}$, traces of $\mathrm{Br}$ and what is the most essential $-20 \mathrm{ppm}$ of $\mathrm{Fe}$ and Co. Such result can be explained by some contamination during the preparation before EDS measurements. However, some possible inhomogeneity of the samples can not be completely excluded and should be kept in mind during the interpretation of the magnetic measurements.

\subsection{Magnetic measurements of clean and copper-modified coals}

Thermomagnetic SQUID measurements of clean and copper-modified carbon coals are given in Fig. 3. The magnetization was measured in two ways: when the samples were cooled without magnetic field (Zero Field Cooled - ZFC), after what the field was turned on and magnetization was measured during heating; and FC Field Cooling, when the magnetization was measured during cooling down with the magnetic field turned on. Fig. 3a represents the magnetic susceptibility of simple purified coal. There are several key features:

- Sharp decrease of magnetic susceptibility near $120 \mathrm{~K}$ on both FC and ZFC curves;

- Paramagnetism at room temperatures;

- Strong paramagnetic contribution increasing at low temperatures.

Starting from the end, strong paramagnetic contribution increasing at low temperatures is typical for carbon materials. It follows the Curie-Weiss law and is obviously connected with number of paramagnetic spins. This number is also proportional to the amount of defects in graphite or graphene-based materials [9] and can be calculated by approximation of the curve by Curie-Weiss law, where Curie constant will be proportional to the amount of paramagnetic spins. In this case, the amount was calculated to be $7.2 \times 10^{18} \mathrm{spins} / \mathrm{g}$. This means that there is one paramagnetic spin per $\sim 7000$ carbon atoms, if only carbon atoms are considered as the source of this signal. 

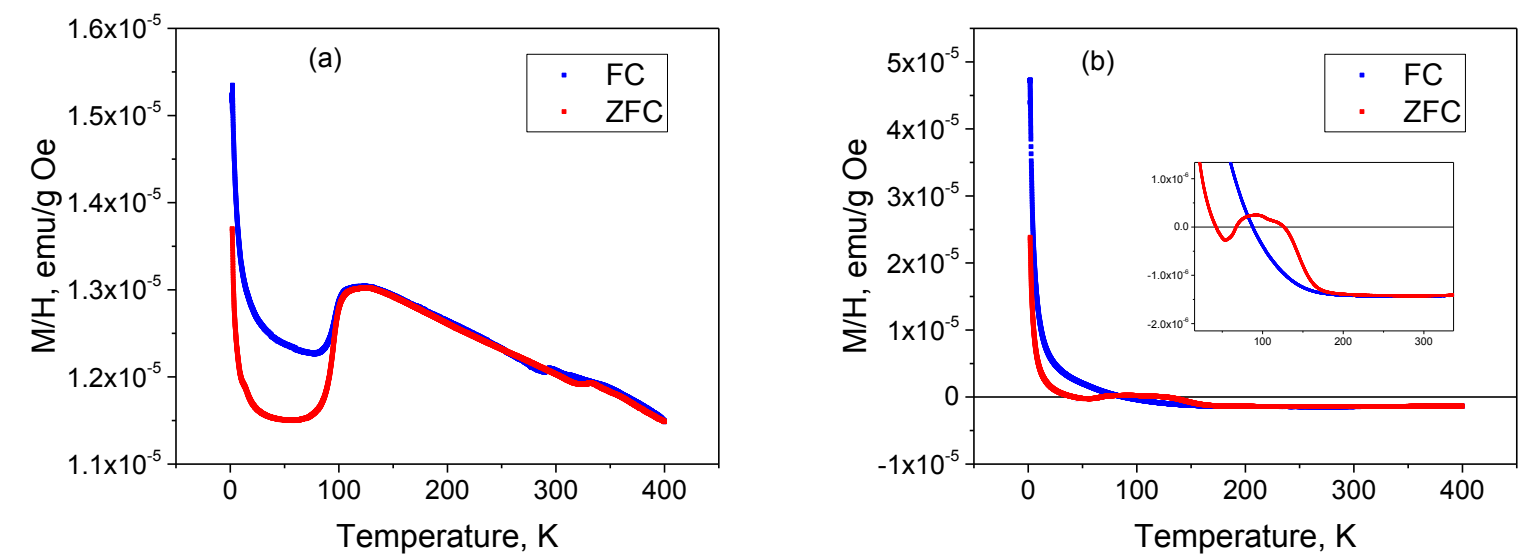

Fig. 3. SQUID FC and ZFC thermomagnetic measurements at 3300 Oe: (a) clean coal; (b) carbon coal modified by copper.

Two other features of magnetic susceptibility of this coal are not typical for carbon materials. While carbon materials usually demonstrate diamagnetic behavior without any sharp changes [9, 10]. Jump of magnetic susceptibility near $120 \mathrm{~K}$ can be interpreted as antiferromagnetic transition. Nature of this effect is debatable. It can be easily explained as a signal from some iron contamination, since iron oxide has similar Verwey transition in the same temperature region. Such explanation is also supported by the shift to paramagnetic region at room temperatures, which is not expected to be a feature of carbon. This can be also a trace of ferromagnetism. However, in our previous work [10] the same jump near $120 \mathrm{~K}$, but without shift to paramagnetic region, was observed for functionalized graphene samples. In that work, carbon origin of this effect was approved by ESR measurements. Thus, the effect can originate from either magnetic contaminations or is connected with carbon magnetic behavior.

In case of modified by copper coal (Fig. 3b), no transitions near $120 \mathrm{~K}$ are observed and the sample is diamagnetic at room temperatures. Nevertheless, increase of paramagnetic signal at $50-170 \mathrm{~K}$ is present. The same phenomena is sometimes observed for different carbon samples [11-13]. Under more precise examination of Fig. $3 \mathrm{~b}$, one can see that this increase is present on ZFC curve and vanishing during cooling on FC curve. This effect can be easily explained by absorbed oxygen, which has antiferromagnetic transition near $50 \mathrm{~K}$. If there is a leakage in SQUID system, or the sample was not completely purged, some oxygen can appear inside measuring area. After oxygen spins are unfrozen at $50 \mathrm{~K}$, they go from antiferromagnetically ordered to paramagnetic state, resulting in increase of paramagnetic contribution. After this, with increasing temperature, oxygen starts to boil and evaporates, resulting in gradual decrease of this effect. If the oxygen has appeared only because of bad purging before measurement, it will be removed by nitrogen flow. The effect of oxygen on magnetic susceptibility was well examined for carbon samples in work [13], using Faraday method. However, usually such effect not discussed at all [11], while in others the similar behavior is called a ferromagnetic-like [12] and possible role of oxygen is not mentioned.

Approximation of low-temperature paramagnetic contribution by Curie-Weiss law gives $2.8 \times 10^{20} \mathrm{spins} / \mathrm{g}$, what is 40 times higher than for the pure coal and probably is explained by copper content, which can be paramagnetic.

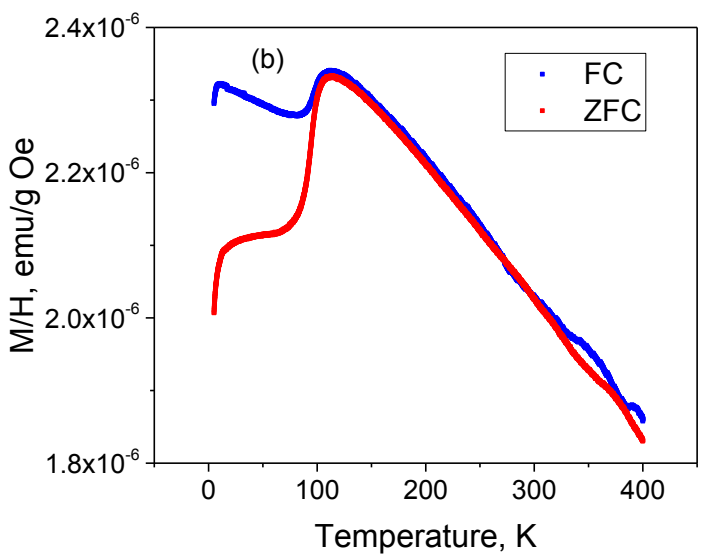

Fig. 4. SQUID FC and ZFC thermomagnetic measurements of dehydrated coal samples at 3300 Oe: (a) clean dehydrated coal; (b) dehydrated carbon coal modified by copper. 


\subsection{Magnetic measurements of dehydrated coals}

In case of dehydrated coals (Fig. 4), in contrast with previous results, antiferromagnetic transition is observed in copper-modified sample, while clear coal demonstrates classical diamagnetic behavior with weaker paramagnetic contributions at low temperatures. No peaks at 50-170 K is observed, indicating that oxygen in previous experiment appeared because of bad purging.

Comparison of the clean non-dehydrated coal and copper-modified dehydrated sample can not be fully adequate, because in copped-modified samples magnetic effects can be dominantly going from copper instead of carbon. However, this can be an argument for the version, that antiferromagnetic transition near $120 \mathrm{~K}$ is connected with magnetic contaminations, which are distributed irregularly and because of this were not detected in fraction, measured by EDS.

One more extraordinary effect is observed in dehydrated carbon coal modified by copper (Fig. 4b). At low temperatures, no paramagnetic contribution is observed. Instead of it, rapid decrease of paramagnetic spins or increase of diamagnetic susceptibility is present. This can be explained as antiferromagnetic ordering of some copper compounds or even strong increase of diamagnetism because of superconductivity.

\section{Conclusion}

A number of amorphous carbon-based materials were investigated by means of SQUID magnetometry. The effect of oxygen on thermomagnetic characteristics was shown. Antiferromagnetic transition was observed for both clean and copper-modified samples. Unfortunately, on the current stage it is not possible to unambiguously connect this effect to either magnetic contaminations or carbon-based magnetism, because there are arguments for both possibilities. According to compositions analysis, up to $20 \mathrm{ppm}$ of $\mathrm{Fe}$ are possible in such material. Rough estimation will give approximately the same value of change of magnetic susceptibility due to antiferromagnetic transition, as going from $20 \mathrm{ppm}$ of $\mathrm{Fe}_{2} \mathrm{O}_{3}$ [12]. However, the similar phenomenon was observed in other carbon samples by ESR [10], indicating that this effect is going from defect-induced carbon paramagnetic spins. Furthermore, observed shift of magnetic susceptibility of carbon coal to paramagnetic region at room temperatures, already needs hundreds $\mathrm{ppm}$ of Fe. Thus, this effect should be examined in more details in any case, either if it is going from contaminations or carbon, to avoid further missteps.

\section{References}

1. Geim, Andre K., and Konstantin S. Novoselov. "The rise of graphene." Nat. mat. 6.3 (2007): 183-191.

2. Červenka, J., M. I. Katsnelson, and C. F. J. Flipse. "Room-temperature ferromagnetism in graphite driven by two-dimensional networks of point defects." Nat. Phys. 5.11 (2009): 840-844.

3. Kiguchi, Manabu, et al. "Magnetic edge state and dangling bond state of nanographene in activated carbon fibers." Phys. Rev. B 84.4 (2011): 045421.

4. Yazyev, Oleg V. "Magnetism in disordered graphene and irradiated graphite." Phys. rev. let. 101.3 (2008): 037203.

5. Schwickardi, Manfred, et al. "Scalable synthesis of activated carbon with superparamagnetic properties." Chem. Comm. 38 (2006): 3987-3989.

6. Faulconer, Emily K., Natalia V. Hoogesteijn von Reitzenstein, and David W. Mazyck. "Optimization of magnetic powdered activated carbon for aqueous $\mathrm{Hg}$ (II) removal and magnetic recovery." J. of Haz. Mat. 199 (2012): 9-14.

7. Arsyad, Nor Amiera Syahida, et al. "Effect of Chemical Treatment on Production of Activated Carbon from Cocos nucifera L.(Coconut) Shell by Microwave Irradiation Method." J. Trop. Resour. Sustain. Sci 4 (2016): 112-116.

8. Babu, V. Suresh, and M. S. Seehra. "Modeling of disorder and X-ray diffraction in coal-based graphitic carbons." Carbon 34.10 (1996): 1259-1265.

9. Sepioni, M., et al. "Limits on intrinsic magnetism in graphene." Phys. Rev. let. 105.20 (2010): 207205.

10. Komlev, A. A., et al. "Magnetism of aniline modified graphene-based materials." J. of Mag. and Mag. Mat. 415 (2016): 45-50.

11. Osipov, V. Yu, et al. "Magnetic and high resolution TEM studies of nanographite derived from nanodiamond." Carbon 44.7 (2006): 1225-1234.

12. Raj, K. Govind, and P. A. Joy. "Magnetism in disordered carbon as a function of the extent of graphitization." S. S. Com. 177 (2014): 89-94.

13. Kawamura, K., Makishima. Y. and Ochiai, Y. "Magnetic susceptibility of oxygen adsorbed on the surface of spherical and fibrous activated carbon." Carbon: Science and Technology. 2(1) (2009): 73-77. 